Z Rheumatol 2010 $69: 107-108$ DOI 10.1007/s00393-009-0568-6

Online publiziert: 29. Januar 2010

๑) Springer-Verlag 2010

\author{
E. Märker-Hermann ${ }^{1}$ • U. Müller-Ladner ${ }^{2}$ \\ ${ }^{1}$ Klinik Innere Medizin IV (Rheumatologie, klinische Immunologie und Nephrologie), \\ HSK Dr. Horst Schmidt Klinik GmbH, Wiesbaden \\ ${ }^{2}$ Abteilung Rheumatologie und klinische Immunologie, Lehrstuhl für Innere Medizin \\ mit Schwerpunkt Rheumatologie der Justus-Liebig-Universität Gießen, \\ Kerckhoff-Klinik Bad Nauheim
}

\title{
Mund, Zahnfleisch, Kiefergelenke und rheumatische Erkrankungen
}

erhöht und bei Rauchern mit einer gesteigerten RA-Inzidenz assoziiert ist [1].

In der auf dem letztjährigen EULARKongress vorgestellten Studie waren die Serumkonzentrationen von Antikörpern gegen zyklische citrullinierte Peptide (CCP-Ak) bei Parodontitispatienten signifikant höher als bei Patienten mit gesundem Zahnfleisch [1]. Die Pathobiologie des Zahnfleisches eröffnet uns hierbei einen neuen Baustein im Verständnis der Zusammenhänge zwischen genetischer Prädisposition („HLA-DRB1 shared epitope"), exogenen Faktoren (Parodontitisbakterien), Durchbrechung der immunologischen Toleranz, Induktion von CCPAk und schließlich Manifestation einer rheumatoiden Arthritis. Das wichtigste Parodontitis-auslösende Bakterium, Porphyromonas gingivalis, exprimiert sein eigenes citrullinierendes Enzym [2]. In diesem Heft der Zeitschrift für Rheumatologie führt Frau PD Dr. Jaqueline Detert in einem umfassenden Übersichtsartikel aus, welche pathogenetischen und epidemiologischen Erkenntnisse bereits jetzt vorliegen und welche spannenden Forschungsprojekte sich aus der Zusammenarbeit zwischen Parodontologie, Mikrobiologie und Immunologie/Rheumatologie ergeben. Praktische Konsequenzen für die tägliche Zahnpflege sowie für die professionelle zahnärztliche Zahnreinigung bzw. Parodontitisbehandlung beim Rheumapatienten werden in dem Beitrag von Prof. Brita Willershausen und Dr. Adian Kasaj dargestellt.
Das zweite wichtige Feld der Kooperation von Rheumatologen und Zahnärzten betrifft das Kiefergelenk. Dem Kinder- und Jugendrheumatologen steht diese Problematik praktisch täglich vor Augen, sind doch bis zu 87\% der Patienten mit juveniler idiopathischer Arthritis (JIA) von einer Kiefergelenkmanifestation betroffen. Da diese häufig schleichend verläuft und bei den betroffenen Kindern zur Destruktion der mandibulären Kondylen führen kann, ist ein aktives diagnostisches Vorgehen erforderlich. Nur eine frühe Diagnose und zeitnahe Therapie kann irreversible Wachstumsstörungen und Gesichtsanomalien verhindern. Dr. Nikolay Tzaribachev stellt zusammen mit Kollegen aus der Zahnheilkunde, Kieferorthopädie und Radiologie in zwei Artikeln die heutigen diagnostischen und therapeutischen Möglichkeiten bei Kiefergelenkarthritis vor. Die Behandlung gründet sich dabei auf medikamentöse, kieferchirurgische und physiotherapeutische Maßnahmen.

\section{7\% der Patienten mit JIA sind von einer Kiefergelenks- manifestation betroffen}

Weitere Themen, die uns in der interdisziplinären Betreuung zwischen Rheumatologie, Zahnheilkunde und auch Kieferorthopädie in besonderer Weise beschäftigen, sind das Sjögren-Syndrom, die systemische Sklerose, die chronisch rekurrierende multifokale Osteomyelitis
Nichtrauchern das Risiko für eine rheumatoide Arthritis (RA) um das 2,6-Fache 
(CRMO) mit Beteiligung der Mandibula, myofasziale Schmerzsyndrome der Temporomandibularregion, die rheumatoide Arthritis mit Kiefergelenkbeteiligung und nicht zuletzt Bisphosphonat-induzierte Osteonekrosen der Kieferknochen.

Wir hoffen daher, dass Ihnen dieser Themenschwerpunkt der Zeitschrift für Rheumatologie zahlreiche neue Anregungen in der Forschung und der klinischen Praxis gibt!

Ihre

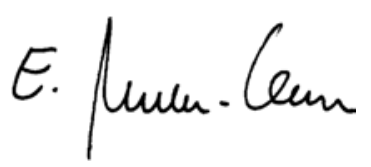

Elisabeth Märker-Hermann

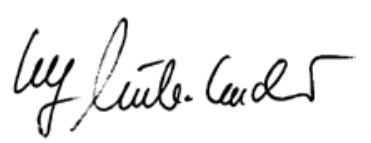

Ulf Müller-Ladner

\section{Korrespondenzadresse}

\section{Prof. Dr. E. Märker-Hermann}

Klinik Innere Medizin IV (Rheumatologie, klinische Immunologie und Nephrologie), HSK Dr. Horst Schmidt Klinik GmbH Ludwig-Erhard-Str. 100, 65199 Wiesbaden elisabeth.maerker-hermann@hsk-wiesbaden.de

\section{Literatur}

1. Molitor JA, Alonso A, Wener MH et al (2009) Moderate to severe adult periodontitis is associated with increased risk of seropositive rheumatoid arthritis in non-smokers: The ARIC study. Ann Rheum Dis 68 (Suppl3):399

2. Lundberg KE (2009) The pathobiology under pinning autoantibodies to citrullinated protein antigens. Ann Rheum Dis 68 (Suppl3):21

\section{Avrion-Mitchison-Preis 2009 für neue Ansätze in der Rheumatherapie verliehen}

Der Avrion-Mitchison-Preis des Deutschen Rheumaforschungszentrums (DRFZ)in Berlin geht in diesem Jahr an den Biowissenschaftler Dr. Kai Kessenbrock (San Francisco), und die Diplom-Biologin Inka Albrecht (Berlin). Die beiden Nachwuchswissenschaftler belegen in Ihren Studien neue Erkenntnisse zu entzündlich-rheumatischenVorgängen und zeigen zukunftsweisende Ansätze für die Rheumatherapie auf. Schwerpunkt ihrer experimentelle Arbeit sind Zellen des bei Rheuma fehlgeleiteten Immunsystems: Anstatt den Körper zu schützen, greifen die Immunzellen Knorpel, Knochen und Gefäße an und rufen damit entzündliche Prozesse hervor, die zu schmerzhaften Beschwerden führen. So zeigte Kessenbrock in Studien an Mäusen, dass so genannte Serinproteasen eine Mittlersubstanz ausschalten, die eine Entzündung verhindern könnte. In einer weitere Studie untersuchte er die Rolle sogenannten Neutrophil Extracellular Traps (NETs), bei der Entstehung von rheumatischen Entzündungen der kleinen Blutgefäßen (Vaskulititen). Die mögliche medikamentöse Hemmung von Serinproteasen und NETs könnte in Zukunft zur Linderung von Rheuma beitragen.

Eine zentrale Funktion für das,immunologische Gedächtnis', und somit das Auftreten von Rheumaschüben haben die T-Helfer-Zellen (Th-Zellen), von denen einige immer wieder das gesunde Gewebe angreifen. Die Unterscheidung,böser' ThZellen von gesunden gelang Preisträgerin Inka Albrecht mithilfe einer Erbgut-Analyse. Das von Ihr entdeckte Gen „Twist1“ ist eines der Gene, das ausschließlich in den entzündungsfördernden Zellen aktiv ist. Die gezielte Ausschaltung der schädlichen Th-Zellen wäre ein bedeutender Fortschritt bei der Bekämpfung von Rheuma.

Der mit 2500 Euro dotierte Avrion-Mitchison-Preis für Rheumaforschung wird seit dem Jahr 2000 zu Ehren des Gründungsdirektors des DRFZ verliehen. Er zeichnet jährlich die beste experimentelle, klinische oder epidemiologische Forschungsarbeit auf dem Gebiet der Rheumatologie aus.
Literatur:

Kessenbrock K, Fröhlich L, Sixt M et al (2008) Proteinase 3 and neutrophil elastase enhance inflammation in mice by inactivating antiinflammatory progranulin. The Journal of clinical investigation 118(7):2438-47

Kessenbrock K, Krumbholz M, Schönermarck et al (2009) Netting neutrophils in autoimmune small-vessel vasculitis. Nature medicine 15(6):623-5

Niesner U, Albrecht I, Janke M et al (2008) Autoregulation of Th1-mediated inflammation by twist1. Journal of Experimental Medicine 205: 1889-1901

Quelle:

Deutschen Rheumaforschungszentrum www.drfz.de 